\title{
A VLSI Implementation of High Sensitive Fingerprint Sensor using Parasitic Insensitive Charge Transfer Circuit
}

\author{
Seungmin Jung \\ School of Information and Telecommunication, Hanshin University, Osan, Korea \\ jasmin@hs.ac.kr
}

\begin{abstract}
This paper implements $80 x 64$ array high sensitive fingerprint sensor with the parasitic insensitive charge transfer circuit. The fingerprint sensor cell uses an active output voltage feedback integrator. The parasitic insensitive charge transfer circuit includes a differential amplifier and two switches to remove parasitic capacitance and transfer charge. The operation is validated by HSPICE for one-pixel and RTL simulation including logic synthesis for the full chip design on condition of $0.18 \mu \mathrm{m}$ typical CMOS process and $1.8 \mathrm{~V}$ power. The voltage difference between a ridge and valley is about $215 \mathrm{mV}$ after 10 clock cycles and $367 \mathrm{mV}$ after 20 clock cycles. The maximum frequency of cell operation is $10 \mathrm{MHz}$. The layout is performed by full custom flow for one-pixel and

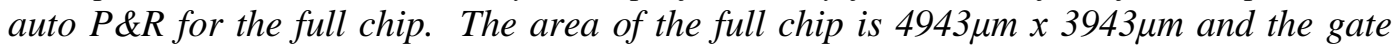

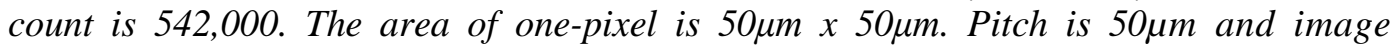
resolution is 508dpi.
\end{abstract}

Keywords: Charge Transfer, Fingerprint Sensor, Parasitic Insensitive, Feedback Integrator, High Sensitive, RTL Synthesis

\section{Introduction}

Fingerprint solution on mobile phone has been got a lot of attention recently as Apple released iPhone6s. Fingerprint sensor can benefits in both on high level of security and also user convenience [1]. Recently, fingerprint sensors are adopted on mobile application environment like a smartphone and tablet. Fingerprint data acquisition can be performed by capacitive sensing circuits [2-7]. To distinguish the capacitance produced between the finger skin and the sensor plate is the original concept of the design for a sensing circuit of the capacitive fingerprint sensors. The detecting circuit for capacitive fingerprint sensor should satisfy the requirements of low power, high sensitivity and wide output dynamic range [6]. Recent works have shown that, by applying capacitive sensing scheme, the fingerprint sensor and its readout circuit can be easily integrated in standard CMOS process. However, the main issue for capacitive sensors concerns parasitic capacitance of the sensor plate, which is formed with the interlayer under the sensor plate. The parasitic capacitance is a critical parameter in conventional sensing circuits because is larger than when a standard dielectric coating film process is used. In general, the thickness of dielectric coating film for protecting the surface of sensor is about $100 \sim 120 \mu \mathrm{m}$. In that case, the difference of capacitance between a ridge and valley is just $0.5 \mathrm{fF}$ to $1 \mathrm{fF}$ at $50 \mathrm{um} \times 50 \mathrm{um}$ pixel size. The value is too small for extracting an image using sensing circuit because the influence of parasitic capacitance is very critical. For low-cost production, parasitic insensitive circuit techniques should be developed.

This paper implements $80 \times 64$ array high sensitive fingerprint sensor with the parasitic insensitive charge transfer circuit. This paper adopts the fingerprint sensor circuit which uses a direct sensing method using an active output voltage feedback integrator [4]. The fingerprint sensor circuit amplifies the sensing signal by 
increasing integration times rather than using an amplifier or a programmable-gainamplifier (PGA), which will improve signal-to-noise ratio (SNR) greatly and allow simple fingerprint driver architecture also. The operation is validated by HSPICE for one-pixel and RTL simulation including logic synthesis for the full chip design on condition of $0.18 \mu \mathrm{m}$ typical CMOS process and $1.8 \mathrm{~V}$ power. The layout is performed by full custom flow for one-pixel and auto P\&R for the full chip.

\section{Parasitic Insensitive Charge Transfer Circuit}

Capacitive sensing devices may suffer from more parasitic components in their touch sensitivity and performance. A capacitive sensor based on charge transfer circuit has also been introduced [5-7]. This paper adopts charge transfer circuit based on the active output voltage feedback integrator. Figure 1 shows the charge transfer integrator based on active output voltage feedback circuit [4]. The finger is simply modeled with a series resistor and a capacitor formed between the finger and a chip surface, $\mathrm{Cf}$. A parasitic capacitor between the sensor plate and isolation metal is represented as $\mathrm{Cp} 1$. The sensor plate is isolated by a metal to prevent the noise from the circuit under the sensor plate as shown in figure 2, which forms a parasitic capacitance between the sensor top metal plate and under metal. Since $\mathrm{C}_{\mathrm{p} 1}$ is relatively large compared to $\mathrm{C}_{\mathrm{f}}$, it should be removed. Some methods for eliminating $\mathrm{C}_{\mathrm{p} 1}$ have been developed [2]. To remove simply $\mathrm{C}_{\mathrm{p} 1}$, the output is applied to the bottom node of $\mathrm{C}_{\mathrm{p} 1}$ to maintain the same potential of the both nodes of $\mathrm{C}_{\mathrm{p} 1}$, which maximizes the sensitivity of the fingerprint sensor. The fingerprint sensor using direct method uses a bezel as a contact to apply signal directly to a finger through it. A signal driven to a finger returns back through the sensor plate. $\Phi 1$ and $\Phi 2$ are the two-phase non-overlapping clocks.
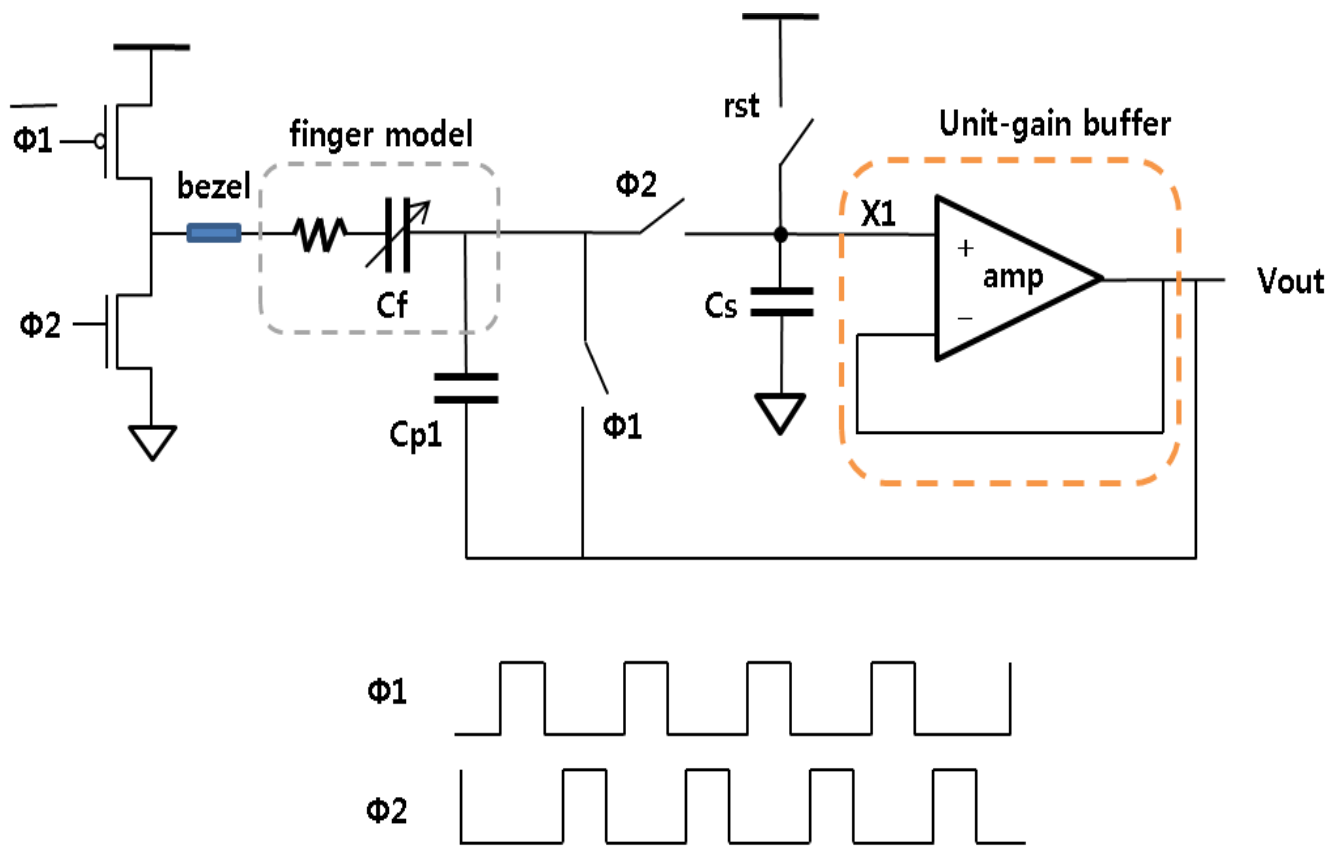

(a) 


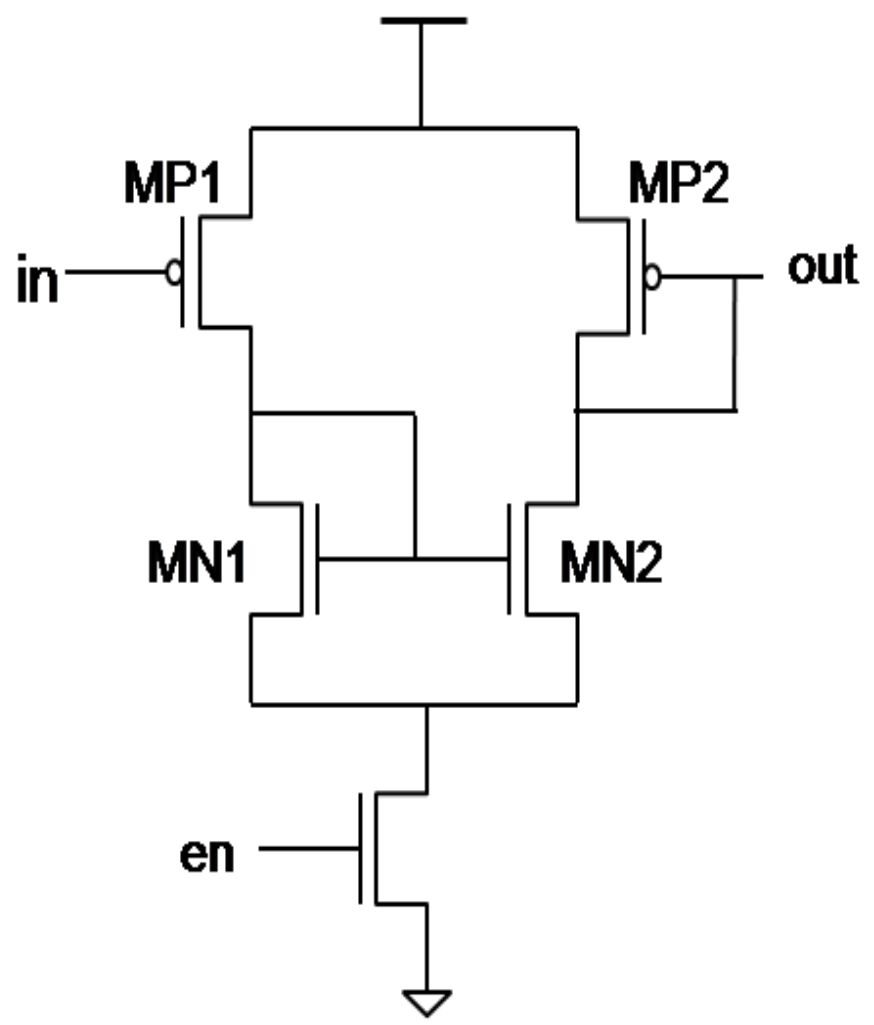

(b)

\section{Figure 1. One Pixel Circuit of Charge Transfer based on Active Output Voltage Feedback Integrator}

The clock signals, $\Phi 1$ and $\Phi 2$, are non-overlap control signal. When $\Phi 1$ is on, two nodes of the parasitic capacitor, $\mathrm{Cp} 1$, are same potential and the influence of $\mathrm{Cp} 1$ is removed. Therefore, the charge of $\mathrm{Cf}$ only is transferred to Cs. The node of negative input of an opamp is virtually shorted to the positive input whose potential is voltage value of capacitor Cs. When $\Phi 2$ is on, two nodes of the parasitic capacitor, Cp1, are same potential as usual because opamp operates like analog buffer. So, the influence of $\mathrm{Cp} 1$ is removed and Vout remains previous state. The accumulated charges in the $\mathrm{Cf}$ are transferred to the output capacitor, Cs. The value of $\mathrm{Cs}$ is $80 \mathrm{fF}$ and same with gate capacitance of opamp. The value of $\mathrm{Cf}$ changes according to ridge and valley of a finger. Figure 1(b) shows the unity-gain buffer, with the signal of en for enabling the unity-gain buffer in the evaluation phase. The role of the unity-gain buffer is tracking the voltage of the node $\mathrm{x} 1$. In case of 50um $x$ 50um pixel size, the difference of $\mathrm{Cf}$ between a ridge and valley is about $0.5 \mathrm{f}$ to $1 \mathrm{f}$, because the thickness of protective coating layer is more than $100 \mu \mathrm{m}$. 


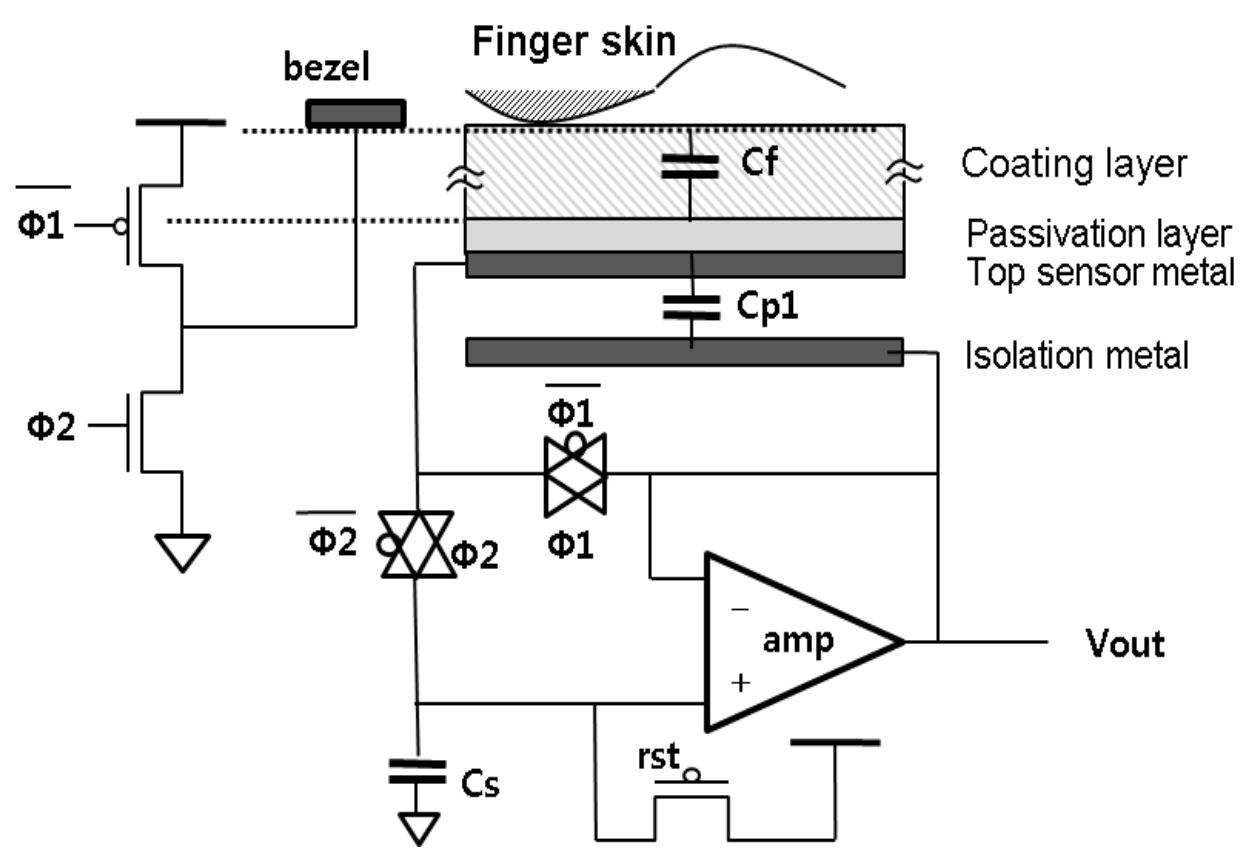

Figure 2. One Pixel CMOS Structure with Hard Coating Layer of Charge Transfer Circuit

Figure 2 shows one pixel CMOS structure with surface protective coating layer of charge transfer circuit. The capacitor formed between the sensor electrode and finger is also modeled. The sensor electrode is separated by the thin passivation layer and coating layer. Therefore, the sensor capacitor, Cf, is composed of two series-connected capacitors which are a capacitor between the electrode and passivation layer and a capacitor between coating layer and the finger skin. Because the thickness of coating layer is larger than passivation, $\mathrm{Cf}$ is almost same capacitor between thick coating layer and the finger skin. The parasitic capacitance of $\mathrm{Cp} 1$ ranges $60 \sim 100 \mathrm{fF}$ which is depend on sensor cell size. The signals generated from the driver are directly fed into the finger through bezel contact. To effectively remove the parasitic capacitor, the isolation metal is connected to the sensor output to maintain the same potential of both nodes of $\mathrm{Cp} 1$.

Figure 3 shows layout of the proposed fingerprint sensor one pixel with the active output voltage feedback integrator using $0.18 \mu \mathrm{m}$ CMOS standard process. The area of one pixel is $50 \mu \mathrm{m} \times 50 \mu \mathrm{m}$ and pitch is $50 \mu \mathrm{m}$ with $508 \mathrm{dpi}$. To confirm the effect of proposed circuit, we extract each parasitic capacitance from the optimized layout of sensor one-pixel. $\mathrm{Cp} 1$ is $78 \mathrm{fF}$. The difference of $\mathrm{Cf}$ between a ridge and valley is $0.5 \mathrm{f}$ to $1 \mathrm{f}$ at protective coating thickness of $100 \mu \mathrm{m}$. In this paper, Cridge is $1 \mathrm{fF}$ and Cvalley is $0.5 \mathrm{fF}$. Functions can be seen by the HSPICE simulation of the cell with condition of $0.18 \mu \mathrm{m}$ typical parameter and $1.8 \mathrm{~V}$ power supply after layout extraction as shown in Figure 3. Figure 4 shows the timing operation at a ridge, valley and between a ridge and valley. The voltage difference between a ridge and valley is about $215 \mathrm{mV}$ after 10 clock cycles and $367 \mathrm{mV}$ after 20 clock cycles. The maximum frequency of cell operation is $10 \mathrm{MHz}$. The simulation results show the parasitic insensitive characteristics which increase touch sensitivity of the circuit. 


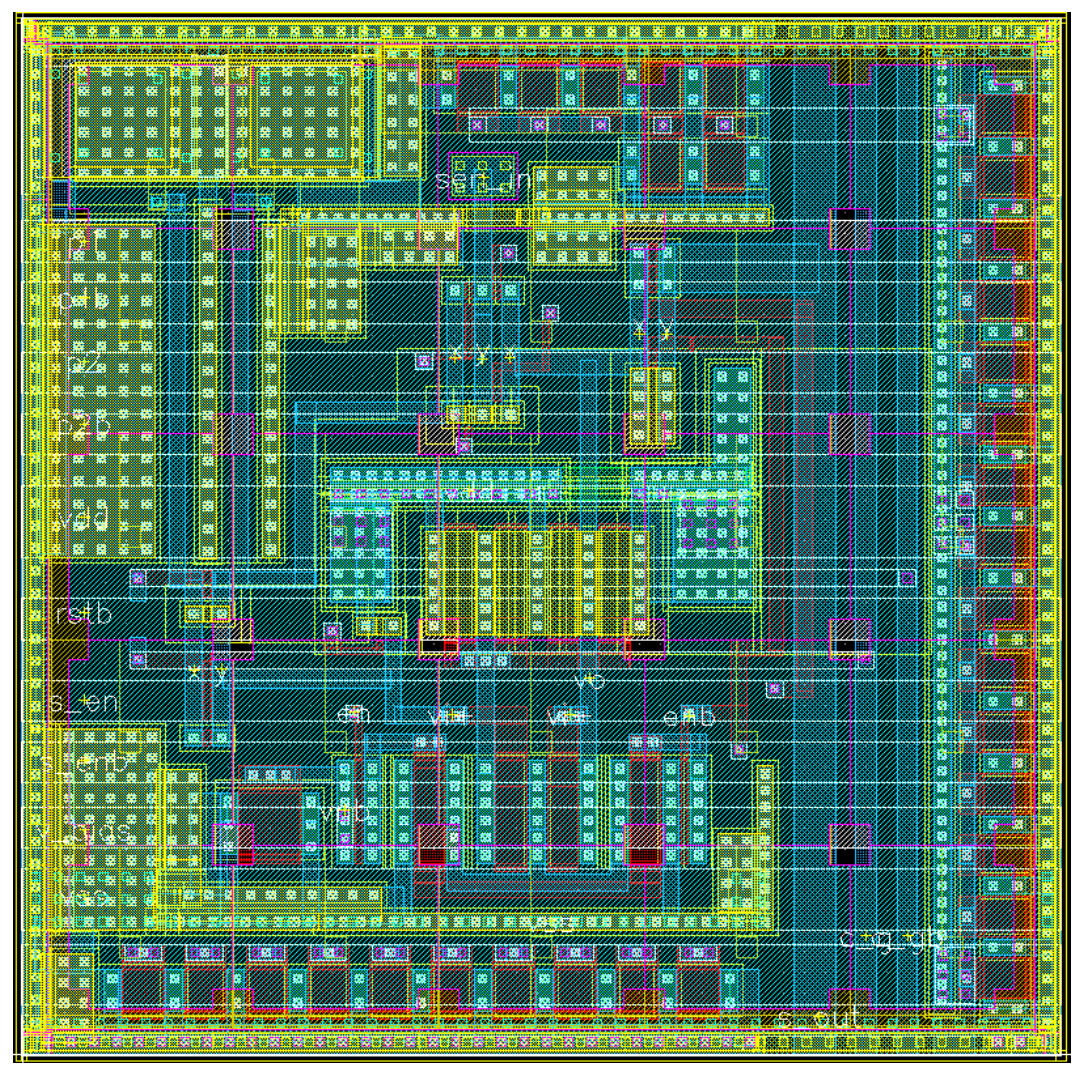

Figure 3. One Pixel Layout (50 $\mu \mathrm{m} \times 50 \mu \mathrm{m}, 0.18 \mu \mathrm{m}$ CMOS Process)

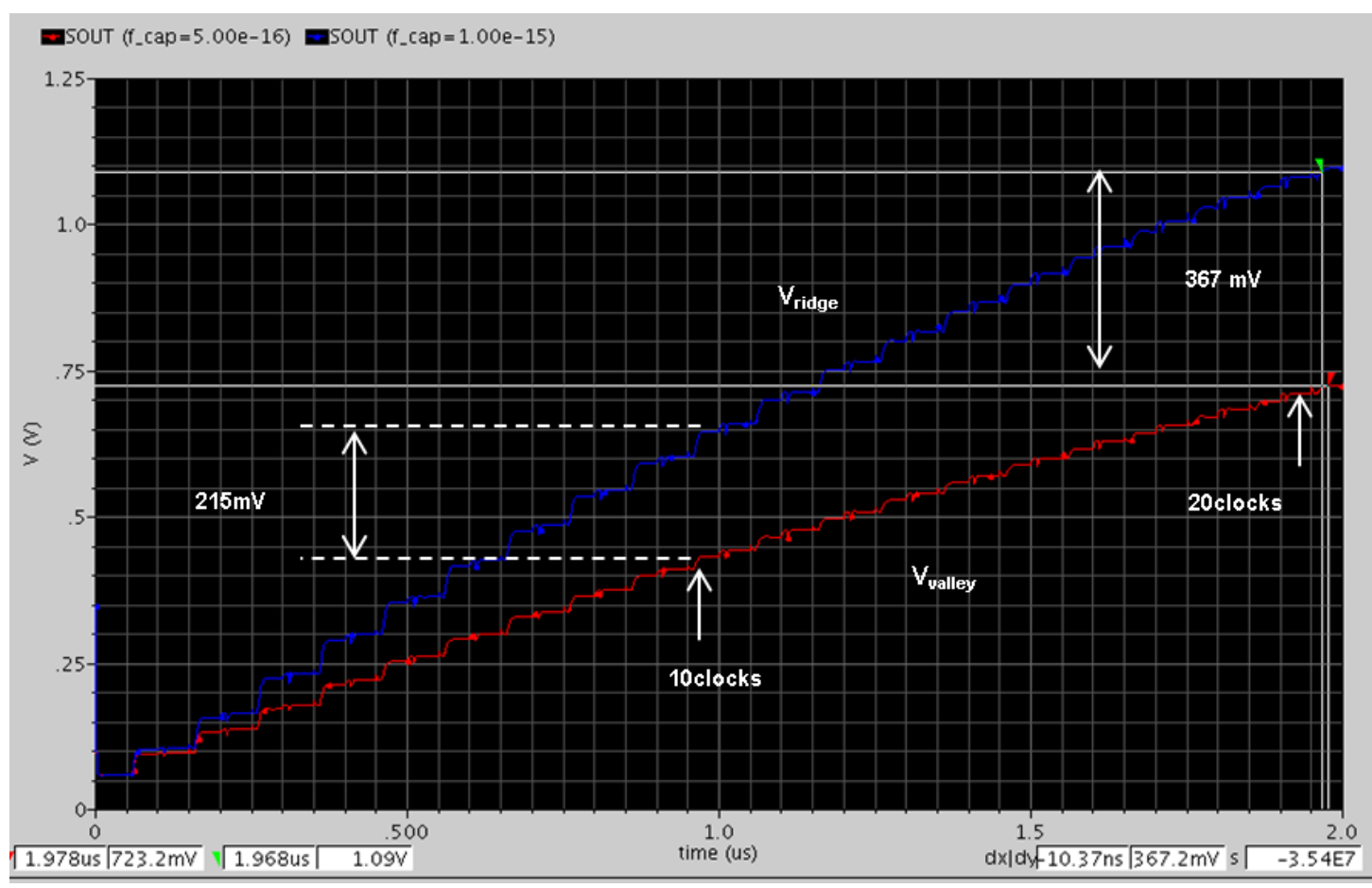

Figure 4. Timing Operation at a Ridge, Valley of One Pixel (0.18um CMOS Process, 1.8V Power, 10Mhz Frequency) 


\section{VLSI Implementation of 80x64 Pixel Array Fingerprint Sensor}

Figure 5 shows the chip block diagram of the proposed area type fingerprint sensor with $80 \times 64$ pixel. The block of variable clock generator generates v_clk signal with variable time duration from maximum 35 clocks to minimum 5 clocks by period signal. $\Phi 1$ and $\Phi 2$ are the two-phase non-overlapping clocks. Figure 6 and 7 show the timing diagram of full chip. The signal STR_ADC means start time of ADC block and EOC means 'end of conversion' after 10 clocks of STR_ADC. Figure 8 shows the logic simulation result of digital block except analog block including sensor array. Figure 9 shows 80 x 64 pixel array chip layout and the area is $4943 \mu \mathrm{m}$ x $3943 \mu \mathrm{m}$ on $0.18 \mu \mathrm{m}$ standard CMOS process. The layout area of one pixel is $50 \mu \mathrm{m} \times 50 \mu \mathrm{m}$ and pixel pitch is $50 \mu \mathrm{m}$. The gate count is 542,000 . The layout of $80 \times 64$ array core cell is performed by full custom design method and the full chip is performed by auto placement and routing of cell based design method.

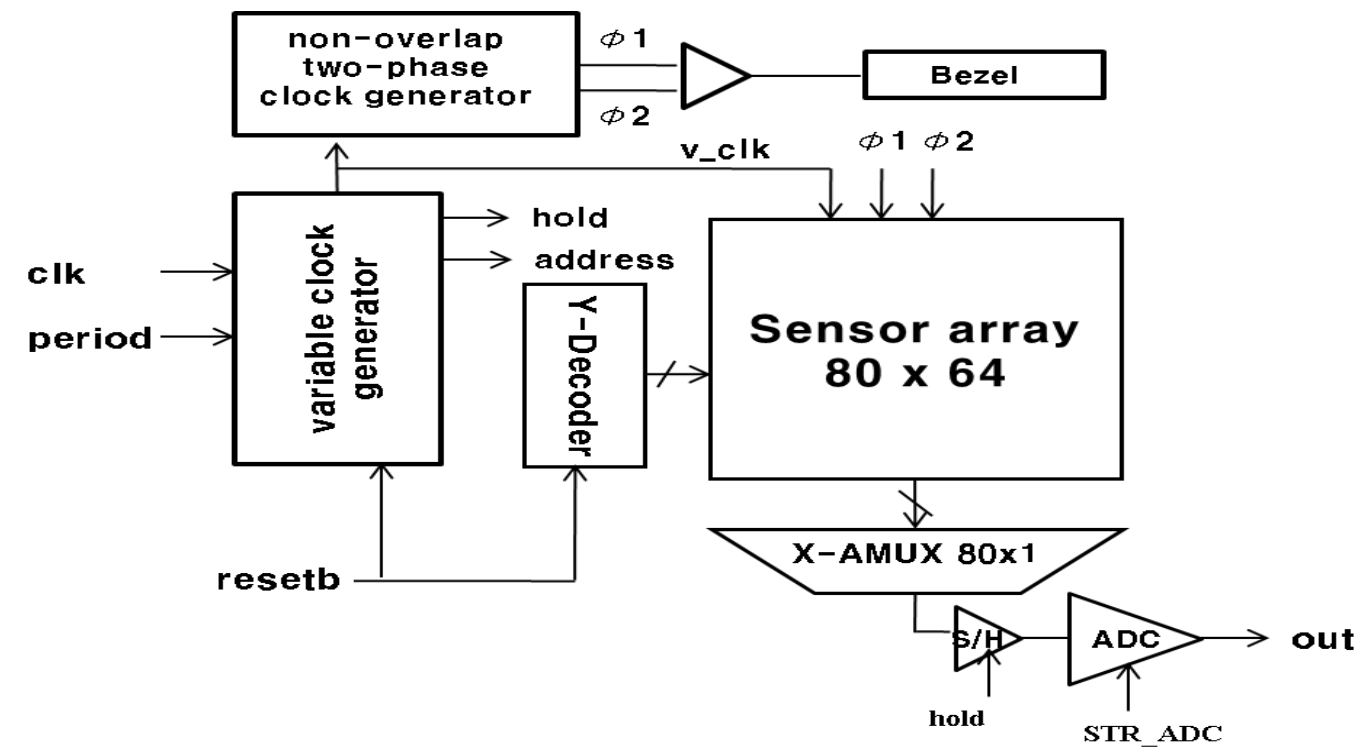

Figure 5. Chip Architecture

clk

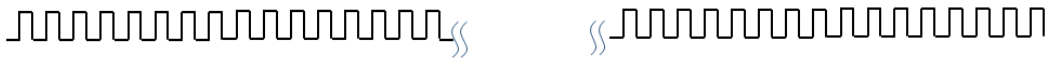

period [3:0]

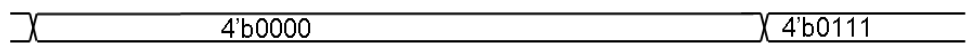

resetb

v_clk

address

hold

cycleb

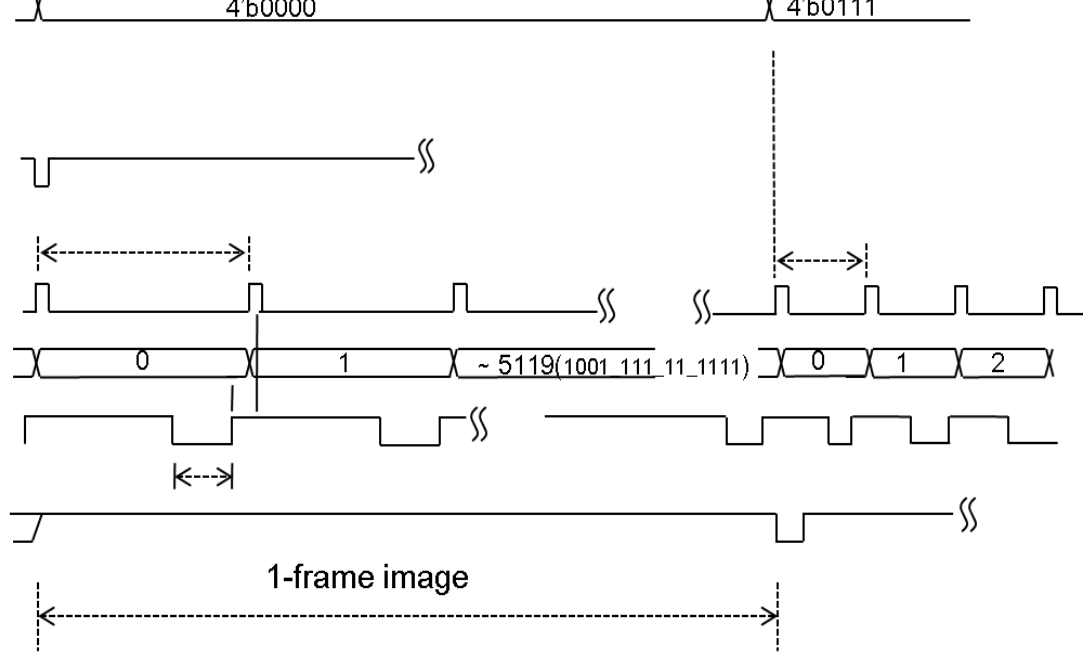

Figure 6. Timing Diagram of Fingerprint LSI 


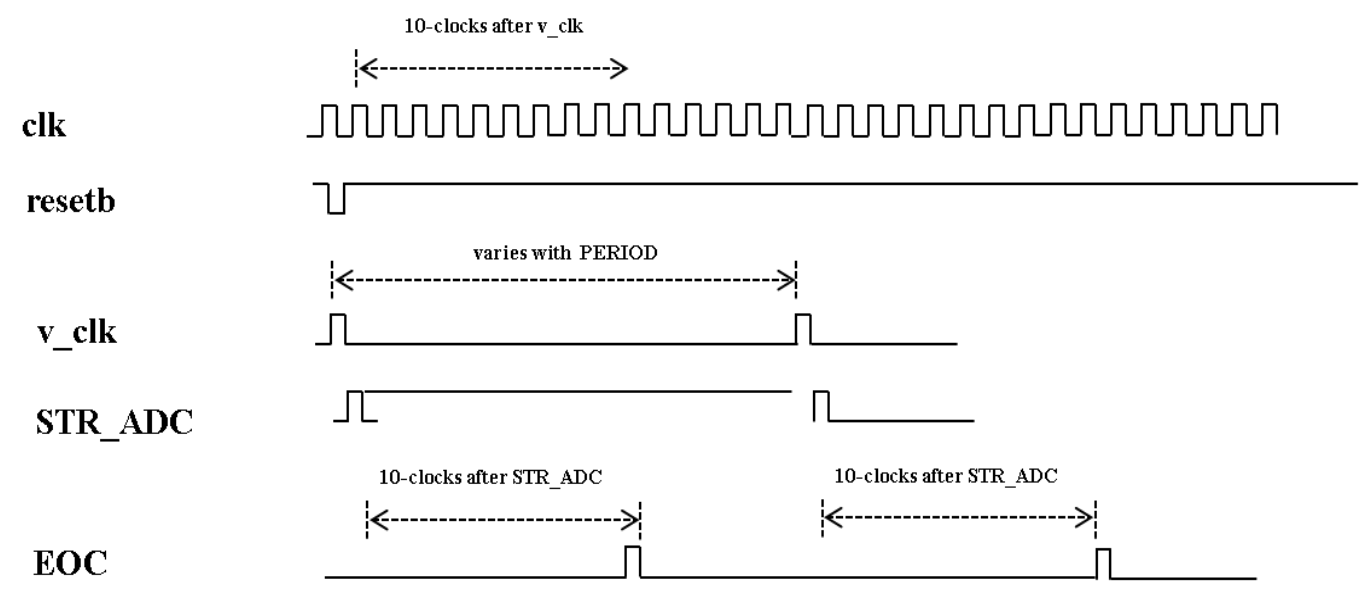

Figure 7. ADC Timing Diagram of Fingerprint LSI

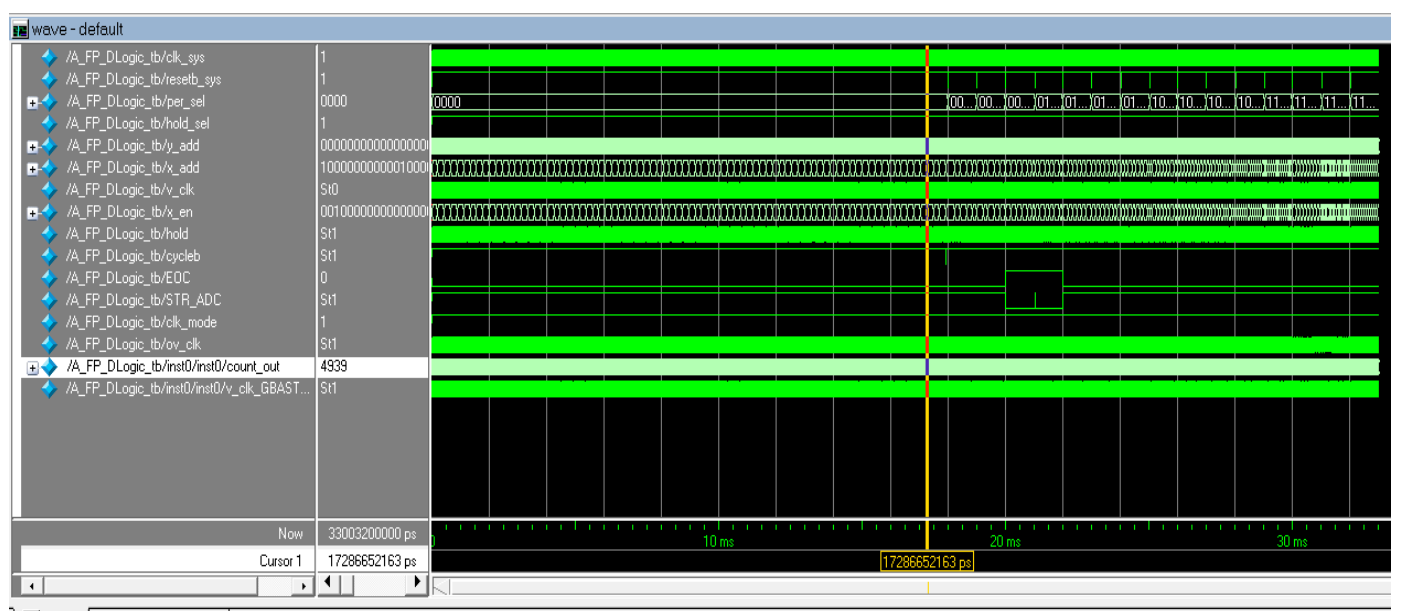

(a)
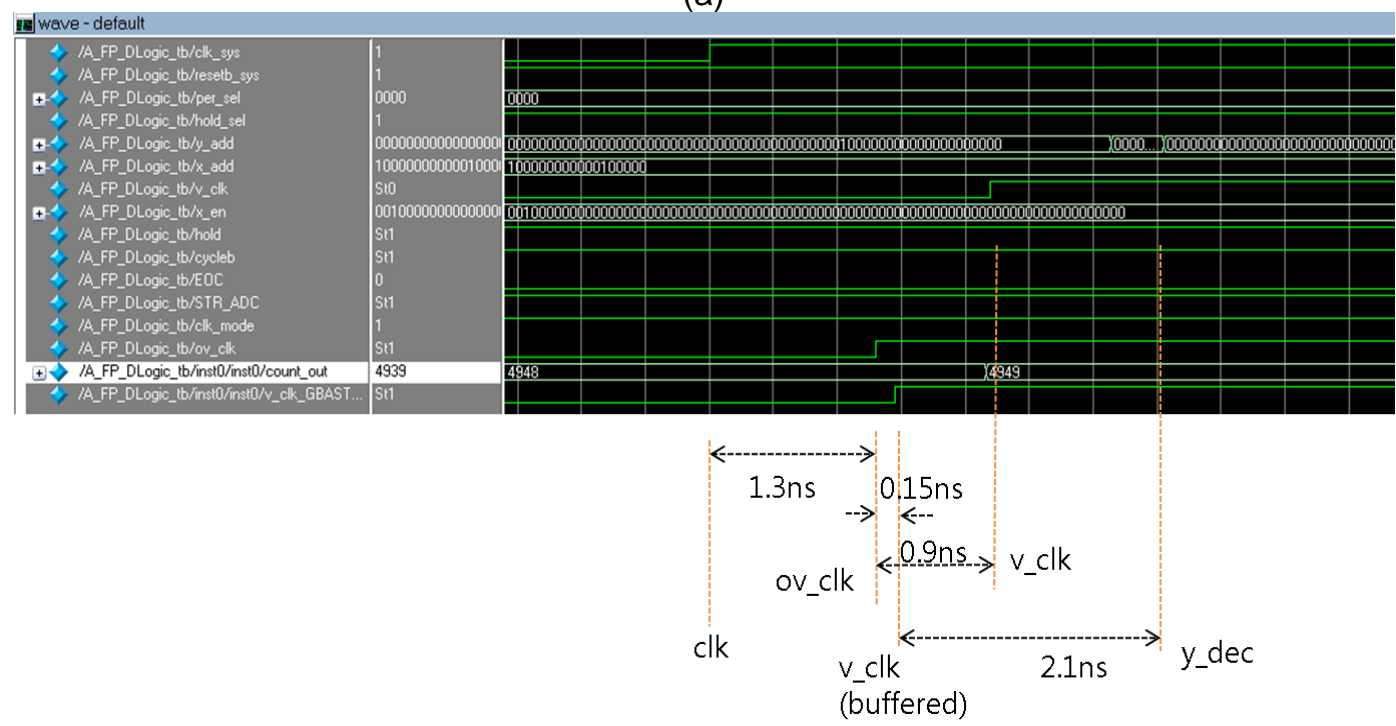

(b)

Figure 8. Logic Simulation Result of Fingerprint LSI 


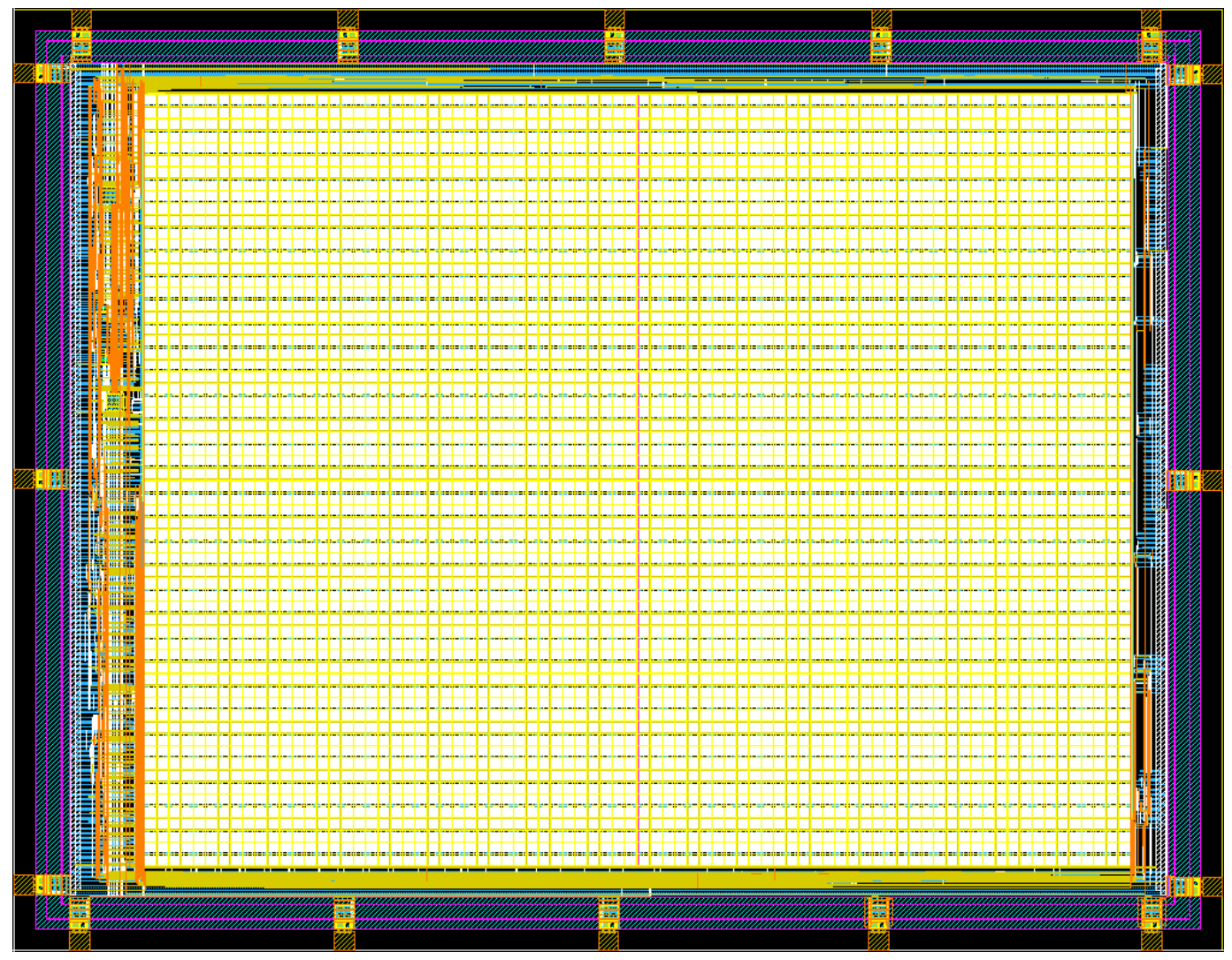

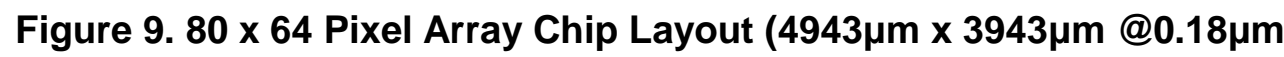
CMOS Process)

\section{Conclusion}

This paper implements $80 \times 64$ array high sensitive fingerprint sensor with the parasitic insensitive charge transfer integrator. The fingerprint sensor cell uses an active output voltage feedback integrator. The detection circuit of one pixel includes a pixel level charge transfer and parasitic insensitive integrator with a differential amplifier with pmos input. A multiple integration scheme is proposed to improve signal-to-noise ratio and amplify the sensing signal, which enables and robust fingerprint sensor driver architecture. The parasitic insensitive charge transfer circuit includes a simple differential amplifier and two switches to remove parasitic capacitance and transfer charge. The operation is validated by HSPICE for one-pixel and RTL simulation including logic synthesis for the full chip design on condition of $0.18 \mu \mathrm{m}$ typical CMOS process and $1.8 \mathrm{~V}$ power. The voltage difference between a ridge and valley is about $215 \mathrm{mV}$ after 10 clock cycles and $367 \mathrm{mV}$ after 20 clock cycles. The maximum frequency of cell operation is $10 \mathrm{MHz}$. The simulation results show the parasitic insensitive characteristics which increase touch sensitivity of the circuit. Full chip logic is synthesized and integrated with 80x64 array sensor core. The layout is performed by full custom flow for one-pixel and auto P\&R for the full chip. The area of the full chip is $19.5 \mathrm{~mm}^{2}(4943 \mu \mathrm{m} \times 3943 \mu \mathrm{m})$ and the gate count is 542,000 . The area of one-pixel is $50 \mu \mathrm{m} \times 50 \mu \mathrm{m}$. Pitch is $50 \mu \mathrm{m}$ and image resolution is $508 \mathrm{dpi}$.

\section{Acknowledgements}

This work was supported by Hanshin University Research Grant. 


\section{References}

[1] M. Gao, X. Hu, B. Cao and D. Li, "Fingerprint sensors in mobile devices", 2014 IEEE 9th Conference on Industrial Electronics and Applications (ICIEA), (2014) June, pp. 1437-1440.

[2] S. M. Jung, J. M. Nam, D. H. Yang and M. K. Lee, "A CMOS integrated capacitive fingerprint sensor with 32-bit RISC microcontroller", IEEE Journal of Solid-State Circuits, vol. 40, (2005), pp. 1745-1750.

[3] J.-C. Liu, Y.-S, Hsiung and M. Lu, "A CMOS Micromachined Capacitive Sensor Array for Fingerprint Detection", IEEE Journal of Solid-State Circuits, vol. 12, no. 5, (2012), pp. 1004-1010.

[4] H. Yeo, "A New Fingerprint Sensor based on Signal Integration Scheme using Charge Transfer Circuit", International Journal of Bio-Science and Bio-Technology(IJBSBT), vol. 7, no. 1, (2015) December, pp. 29-38.

[5] S.-M. Jung, "Active Capacitive-Sensing Circuit Technique for Image Quality Improvement on Fingerprint Sensor", Journal of Next Generation Information Technology (JNIT), vol. 4, no. 3, (2013), pp. 47-53.

[6] H. Yeo, "A Parasitic-Insensitive Charge Transfer Circuit for Capacitive Sensing using Active Output Voltage Feedback Technique", Journal of Next Generation Information Technology(JNIT), vol. 4, no. 5, (2013) July, pp. 164-171.

[7] S. Min Jung, "An Implementation of Parasitic Insensitive 128 x100 Pixels Fingerprint Sensor using Modified Switched Capacitor Integrator", International Journal of Bio-Science and BioTechnology(IJBSBT), vol. 6, no. 6, pp. 121-128, (2014) December.

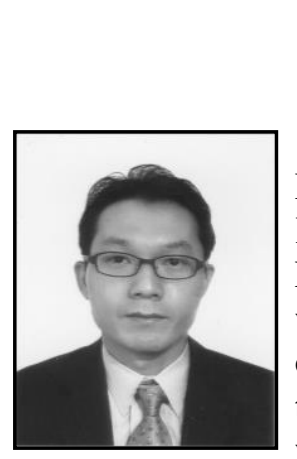

\section{Author}

Seungmin Jung, he received the BS, MS and Ph.D. degrees in Department of Electronic Engineering from Yonsei University, Seoul, Korea, in 1990, 1992 and 2006. From 1992 to 1997, he was Senior Engineer of the ASIC Division at Samsung Electronics Co., Ltd, where he worked on the design of memory compiler. He also worked on the design of mixed signal VLSI. From 1998 to 2006, he joined the faculty of Yongin-Songdam University, Yongin, Korea, where he was assistant professor in the Information and Communication Department. In 2006, he joined the faculty of Hanshin University, Osan, Korea, where he is currently professor in the School of Information and Telecommunication. His research interests include biometric CMOS sensors, SoC design and high-speed interface mixed circuit design. (email: jasmin@ @hs.ac.kr) 
International Journal of Smart Home

Vol. 9, No. 7 (2015) 